Revue musicale OICRM

revue musicale oicrm

\title{
"Panel sur la critique musicale ", dans le cadre du colloque international Qu'en est-il du goût musical dans le monde au XXIe siècle ?, 28 février 2013, Faculté de musique, Université de Montréal, salle Serge-Garant
}

\section{Caroline Marcoux-Gendron}

Volume 3, numéro 1, 2016

URI : https://id.erudit.org/iderudit/1060125ar

DOI : https://doi.org/10.7202/1060125ar

Aller au sommaire du numéro

Éditeur(s)

OICRM

ISSN

2368-7061 (numérique)

Découvrir la revue

Citer cette note

Marcoux-Gendron, C. (2016). « Panel sur la critique musicale ", dans le cadre du colloque international Qu'en est-il du goût musical dans le monde au XXIe siècle ?, 28 février 2013, Faculté de musique, Université de Montréal, salle Serge-Garant. Revue musicale OICRM, 3(1), 137-146. https://doi.org/10.7202/1060125ar
Résumé de l'article

Dans le cadre du colloque Qu'en est-il du goût musical dans le monde au XXIe siècle ? présenté à la Faculté de musique de l’Université de Montréal du 28 février au 2 mars 2013, un panel a réuni les critiques musicaux Renaud Machart (France), Anne Midgette (É.-U.) et André Péloquin (Québec, Canada) autour de la question « En quoi l'exercice de la critique musicale participe-t-il à l'édification du goût musical des lecteurs-auditeurs ? ". Une série d'enjeux a été abordée au cours de cet échange, dont les objectifs de la critique musicale, son influence sur les lecteurs-auditeurs, mais aussi les obligations éthiques du critique et l'avenir de cette pratique. Les points de vue des trois participants se sont révélés différents à bien des égards, à l’image de la diversité des genres musicaux dont ils traitent, de leurs parcours professionnels respectifs ainsi que des contextes géographiques au sein desquels ils œuvrent. 


\title{
"Panel sur la critique musicale ", dans le cadre du colloque international Qu'en est-il du goût musical dans le monde au XXI siècle?, 28 février 2013, Faculté de musique, Université de Montréal, salle Serge-Garant
}

\author{
Caroline Marcoux-Gendron
}

\begin{abstract}
Résumé
Dans le cadre du colloque Qu'en est-il du goût musical dans le monde au XXI' siècle? présenté à la Faculté de musique de l'Université de Montréal du 28 février au 2 mars 2013, un panel a réuni les critiques musicaux Renaud Machart (France), Anne Midgette (É.-U.) et André Péloquin (Québec, Canada) autour de la question «En quoi l'exercice de la critique musicale participe-t-il à l'édification du goût musical des lecteurs-auditeurs ? ». Une série d'enjeux a été abordée au cours de cet échange, dont les objectifs de la critique musicale, son influence sur les lecteurs-auditeurs, mais aussi les obligations éthiques du critique et l'avenir de cette pratique. Les points de vue des trois participants se sont révélés différents à bien des égards, à l'image de la diversité des genres musicaux dont ils traitent, de leurs parcours professionnels respectifs ainsi que des contextes géographiques au sein desquels ils œuvrent.
\end{abstract}

Mots clés : critique musicale ; goût ; lecteurs-auditeurs ; discours sur la musique ; réception musicale.

\begin{abstract}
At the symposium Qu'en est-il du goût musical dans le monde au XXI siècle? (What has become of musical taste in the world in the 21st century?) held at the Faculty of Music of the University of Montreal from February 28 to March 2, 2013, a panel brought together music critics Renaud Machart (France), Anne Midgette (Us) and André Péloquin (Quebec, Canada) around the question "How has the act of music criticism participated in the edification of the musical taste of readers/listeners?" Addressed during this exchange was a series of issues including the goals of musical criticism, its influence on readers-listeners, but also the ethical obligations of the critic, and
\end{abstract}


the outlook for this activity. The three panelists' viewpoints differed on many levels, reflecting the diversity of the musical genres they cover, of their respective professional trajectories, and of the geographical contexts within which they work.

Keywords: music criticism; taste; readers-listeners; discourse about music; music reception.

La critique musicale tantôt fascine, tantôt dérange, mais toujours revêt un caractère insaisissable ; s'agit-il d'un art, d'une science, d'un métier ? (Berthet 2006, p. 67-68). Dans le cadre du colloque sur l'esthétique musicale intitulé Qu'en est-il du goût musical dans le monde au $X X I^{e}$ siècle ? et présenté à la Faculté de musique de l'Université de Montréal du 28 février au 2 mars 2013, un panel a réuni trois critiques musicaux d'horizons différents. Renaud Machart (France), Anne Midgette (États-Unis) et André Péloquin (Québec, Canada) se sont intéressés à la question « En quoi l'exercice de la critique musicale participe-t-il à l'édification du goût musical des lecteurs-auditeurs? » et ont répondu aux interrogations du public venu nombreux pour l'occasion. L'échange s'est articulé à la fois autour d'aspects pragmatiques - les genres musicaux traités, les médias à travers lesquels s'exprime le critique, les « objets » discutés (disque, concert, etc.), le public visé - et de thèmes en appelant à une vision plus personnelle des participants, notamment les objectifs de la critique musicale et son influence sur les lecteurs-auditeurs.

D'entrée de jeu, le président de séance Michel Duchesneau, musicologue dont les travaux portent notamment sur la critique musicale en France au $\mathrm{xx}^{\mathrm{e}}$ siècle, a orienté cette réflexion collective en identifiant des enjeux de la pratique ayant traversé les époques : la disparition des repères esthétiques, l'autonomie artistique de l'œuvre, sa pertinence sociale qui supplanterait désormais les considérations esthétiques et historiques, la nature du discours sur l'œuvre et la crainte du jugement erroné.

Le bilan de la critique musicale d'hier à aujourd'hui ainsi esquissé a lancé le débat quant à l'avenir de cette pratique. En somme, il semble que peu de réponses font consensus, à l'image de la nature souvent controversée de la critique musicale.

\section{PRÉSENTATION DES PARTICIPANTS}

L'un des principaux intérêts de ce panel résidait dans la diversité des profils des trois participants. Le premier, Renaud Machart, est un critique musical notamment actif dans la presse écrite française (Le Monde, par exemple) ; il détient une formation en musique au Conservatoire de Tours et en musicologie à l'Université de Tours. À la fois directeur de festivals, animateur et producteur radio, il est l'auteur de plusieurs ouvrages, entre autres sur John Adams, Leonard Bernstein, Francis Poulenc et Stephen Sondheim. Anne Midgette, diplômée de 1'Université Yale en Classical Civilization, est, quant à elle, actuellement chef critique pour la musique classique au Washington Post (É.-U.), après des débuts sur le continent européen. Au cours de sa carrière, elle a collaboré à de nombreux périodiques (The Wall Street Journal, The New York Times, Opera News, The Los Angeles Times) et coécrit les ouvrages The King and I sur Luciano Pavarotti et My Nine Lives sur le pianiste Leon Fleischer. Finalement, André Péloquin, formé en littérature comparée à l'Université de Montréal, est plutôt actif dans le 
domaine de la musique populaire. Il a été chef critique de la section " musique " au journal Voir (août 2012-janvier 2015) et agit à la fois comme chroniqueur, journaliste, réalisateur et recherchiste indépendant (voir son blogue).

\section{LES GRANDS AXES DU PANEL}

Lors du premier tour de table où chaque panéliste a exprimé sa vision de la critique musicale à l'heure actuelle, peu de recoupements ont été notés dans les témoignages étant donné la variété de genres musicaux dont traitent ces critiques et les différents moyens de diffusion qu'ils utilisent. Aux côtés du traditionnel journal se trouvent maintenant les blogues dont Péloquin et Midgette font fréquemment usage, les sites Internet spécialisés comme Pitchfork ${ }^{1}$, les agrégateurs de critiques tels que Metacritic, ou encore la radio, moyen de diffusion que Machart connaît en tant qu'animateur de l'émission " Le Mitan des musiciens »- nommée «Le Matin des musiciens » au moment du panel en 2013 - sur France Musique ${ }^{2}$ et que Péloquin a fréquenté comme étudiant à l'Université de Montréal. Pour chaque média prévaut une approche différente en termes de niveau de langue, de longueur des interventions, de fréquence des publications, ce qui modifie le contact du critique avec les lecteurs-auditeurs. Par exemple, Machart affirmait que la publication dans un journal revêt un caractère impersonnel, ce qui l'a éventuellement amené à s'interroger sur les véritables destinataires de ses écrits. À l'opposé, la radio lui permet un rapide retour des auditeurs, tout comme elle offre la possibilité de diffuser les œuvres musicales discutées. Chez Midgette, le blogue s'affirme comme un lieu d'échanges constants avec ses lecteurs, et cette notion de dialogue avec le public est apparue au cœur de ses préoccupations. À ce propos, le docteur en esthétique et sciences de l'art Dominique Berthet écrit d'ailleurs que " [1]e discours critique est en effet un jugement destiné à l'autre, qui crée une relation avec autrui » (2006, p. 106). Or, la question du rapport au lecteurauditeur se pose en de nombreux autres termes : dans le cas d'un concert, s'adresset-on à ceux qui étaient présents ou aux absents? Vise-t-on les connaisseurs ou les néophytes? À cet égard, il est intéressant de noter que si le critique peut être considéré comme un " auditeur particulier »(Griffiths 2004, p. 1063), aucune formation ni aucun profil précis ne prédominent. Les exemples contrastants de Machart, musicien et musicologue de formation, et de Péloquin, autodidacte prônant une approche plus instinctive, illustrent cette réalité.

1 Portail américain dédié essentiellement à la musique indie. Cette plateforme se démarque par des critiques musicales au style singulier, dont la longueur et le ton se détachent souvent des normes journalistiques. Voir http:// pitchfork.com (consulté le 14 octobre 2015).

2 L'activité de Machart à la radio ne relève pas précisément de la critique musicale, mais ce média n'en demeure pas moins un canal de diffusion possible pour une telle activité. 


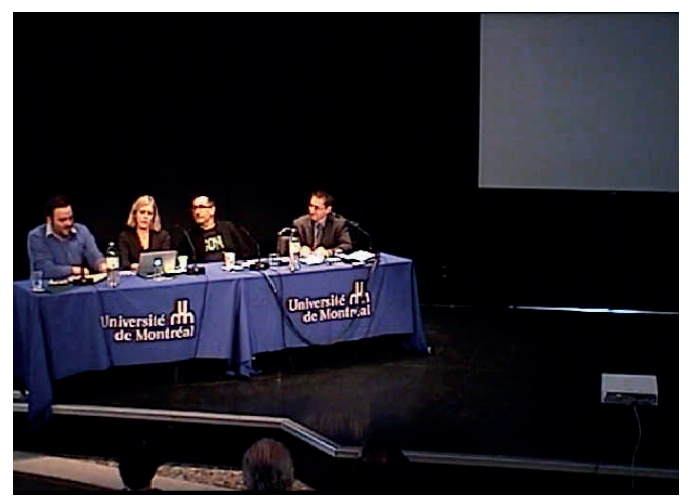

Extrait vidéo 1: André Péloquin et Renaud Machart à propos de leurs parcours.

Le critique musical Paul Griffiths souligne d'ailleurs que ce sont l'expérience et la perspicacité bien plus que les devoirs « faits la veille » qui déterminent la compétence dans ce domaine (2004, p. 1067).

Sur le plan des objectifs de la critique, les points de vue des panélistes ont demeuré divergents. Midgette a insisté sur le développement du sens critique de ses lecteurs, soulignant toutefois que sa démarche change lorsqu'il s'agit de musique contemporaine, où elle adopte une approche plus pédagogique visant à initier son lectorat aux compositeurs, aux œuvres, etc. Chez Machart, il a plutôt été question de la critique comme d'un moyen de documenter, de laisser une mémoire par l'entremise d'une sorte de compte rendu.

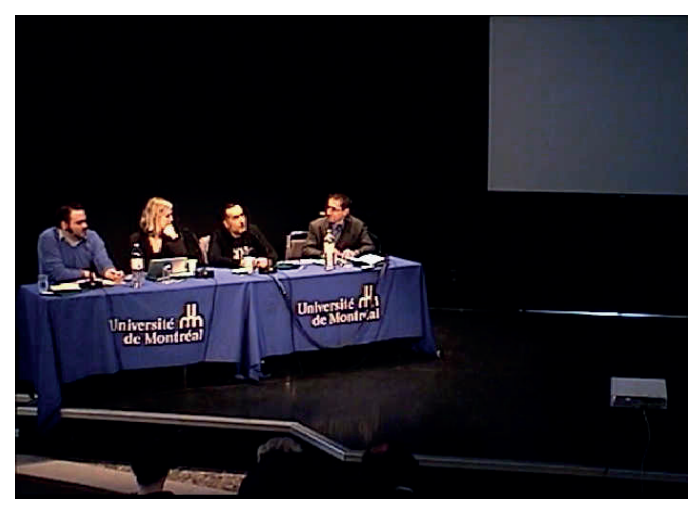

Extrait vidéo 2: Renaud Machart sur le rôle de mémoire joué par la critique musicale.

Plusieurs auteurs traitent d'ailleurs de cette fonction historique de la critique qui agit a posteriori, comme témoignage de la réception des œuvres (Berthet 2006 ; Duchesneau 2007 ; Molino 2006). Finalement, Péloquin a traduit sa relation aux lecteurs par une analogie avec le métier de disquaire, qui fait des suggestions à ses clients. Selon le panéliste, cette comparaison n'est pas sans évoquer l'aspect économique omniprésent dans les médias culturels d'aujourd'hui. Bref, il apparaît que la critique peut adopter différentes formes ou " postures ", pour paraphraser Duchesneau en ouverture du panel. Or, qu'en est-il maintenant de son réel impact sur le lecteur-auditeur? 
À ce compte, Péloquin a souligné que la critique musicale peut agir sur la vente de disques lorsque les commentaires élogieux sont repris par des publicitaires. Il considère également qu'elle aide à façonner le goût des mélomanes, opinion qu'a réfutée Midgette qui juge au contraire son pouvoir très mince et note un désintérêt marqué pour la critique à 1'heure actuelle ${ }^{3}$. Autre son de cloche du côté de Machart qui a dit emprunter au compositeur et auteur américain Ned Rorem la phrase choc voulant que la critique ne serve qu'à "donner une opinion à ceux qui n'en ont pas ». Le panéliste français a affirmé trouver un plaisir "vaniteux " à aider à remplir les salles, mais a aussi rappelé que la critique n'est pas une science, en témoignent les opinions divergentes ayant trait à une même œuvre. Finalement, Machart, dont le style est ouvertement incisif, a souligné que seules les très bonnes et les très mauvaises " notes » sont lues, faisant écho à Griffiths pour qui « la critique la plus efficace n'est jamais très loin de l'inconvenance » (2004, p. 1063).

Parmi les questions du public, les considérations éthiques ont été soulevées plusieurs fois et les réponses se sont faites très différentes selon le panéliste. En Europe, les invitations à des concerts et les voyages payés pour les critiques sont chose courante, tandis qu'on les interdit en Amérique. Les intervenants français et américain s'opposaient également en ce que Machart a dit écrire sur ses amis compositeurs - ce qui ne l'empêche pas d'exprimer ce qu'il pense, a-t-il précisé - tandis que Midgette y voyait une infraction à son code d'éthique.

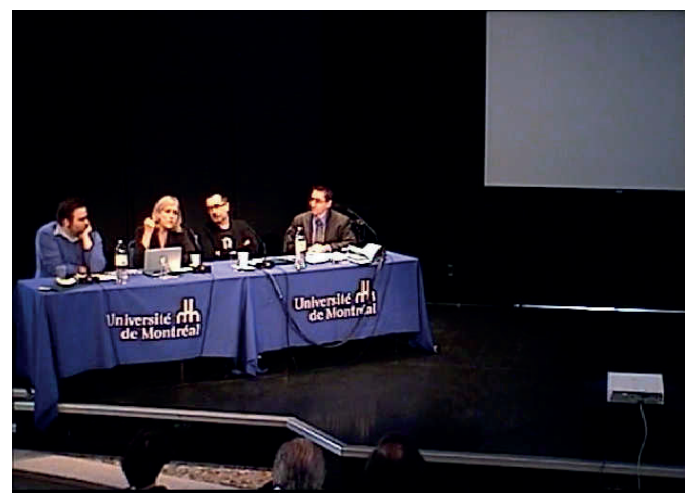

Extrait vidéo 3 : Anne Midgette et Renaud Machart sur l'éthique du critique.

Péloquin a, quant à lui, souligné un problème inhérent au « microcosme » québécois où les gens sont peu portés sur les débats relevés. La proximité entre artistes et critiques les premiers pouvant facilement entrer en contact avec les seconds, notamment grâce aux réseaux sociaux - induirait à son avis un climat où personne n'ose froisser un confrère.

Ainsi, la critique musicale est abordée de manière différente selon le pays, et Midgette considère d'ailleurs que le rapport économique entre un État et son milieu artistique joue un rôle à cet égard. Plus précisément, elle a expliqué que dans un pays

3 Midgette a donné l'exemple de son mari qui, en tant que professeur à Juilliard, a dû changer le titre de son cours Music Criticism pour Writing About Music étant donné le peu d'enthousiasme des étudiants. 
où le financement étatique des arts est important, la critique serait une sorte de reflet de l'utilisation des fonds publics, contrairement aux États-Unis par exemple, où le milieu artistique est peu subventionné et où la critique apparaît plutôt comme un service d'orientation - de ce qui vaut la peine d'être vu et entendu. Ces considérations mettent en lumière la diversité des paramètres qui régissent la pratique de la critique musicale et ces trois témoignages révèlent une multiplicité de profils et de positions, peut-être aussi grande que la somme des individus qui s'y adonnent finalement.

\section{LES ENJEUX DE LA CRITIQUE MUSICALE, D'HIER À AUJOURD'HUI}

Malgré la nature très libre de la discussion, le programme exposé par Duchesneau en ouverture de séance a sous-tendu l'ensemble du panel. Il s'agissait de comprendre les enjeux qui traversent l'exercice de la critique musicale, d'abord par un survol de la pratique d'hier à aujourd'hui, pour ensuite réfléchir à son avenir.

La disparition des repères esthétiques au sein d'une même culture tenait lieu de premier enjeu, et le critique Péloquin a corroboré cette idée en parlant d'un actuel " chaos du jugement » étant donné la prolifération des plates-formes qui permettent à tous d'émettre leur opinion. L'abondance et l'accessibilité de l'offre musicale décupleraient d'ailleurs le phénomène en retirant aux critiques « officiels » la primeur des œuvres. Cette réalité a par ailleurs semblé plus marquée pour Péloquin qui travaille dans le domaine de la musique populaire et qui est très familier avec les médias électroniques.

Le deuxième enjeu était celui de l'autonomie artistique de l'œuvre qui empêcherait le recours à des principes esthétiques partagés. Cette idée n'a pas été approfondie notamment parce que les panélistes ont évité la question des critères d'évaluation d'un jugement critique. Du peu qui a été dit sur le milieu de la création musicale, rien ne portait sur l'existence, ou plutôt l'absence de principes esthétiques communs aux œuvres contemporaines.

Le troisième enjeu concernait la pertinence sociale de l'œuvre qui supplanterait toute autre forme de considération, esthétique ou historique. Midgette s'est prononcée sur la question, soulignant que les gens s'identifient particulièrement à la musique qu'ils écoutent et se définissent à travers elle. La panéliste croit d'ailleurs que la difficulté observée en musique classique pour le renouvellement des publics relèverait de l'image élitiste qu'on lui attribue généralement ${ }^{4}$.

En ce qui concerne la nature du discours sur l'œuvre (quatrième enjeu), Péloquin a fourni un intéressant témoignage en s'appuyant sur l'exemple du site Pitchfork. Les critiques verbeuses, qui glorifient les œuvres autant qu'elles les détruisent, y font de la musique le prétexte d'un discours devenu le véritable centre d'intérêt. La figure de Lester Bangs, critique américain au style sans pareil et dont les écrits font l'objet de recueils (Bangs 1988, 2003), incarnerait, d'après le panéliste québécois, ce « culte de

4 Cette question riche a suscité l'attention de plusieurs chercheurs déjà, notamment en regard du public de jeunes adultes. Voir, entre autres, Kolb pour le contexte anglo-saxon $(2000,2001)$ et Oliveira-Menezes pour le contexte montréalais (2015). 
la personnalité » entourant certains critiques.

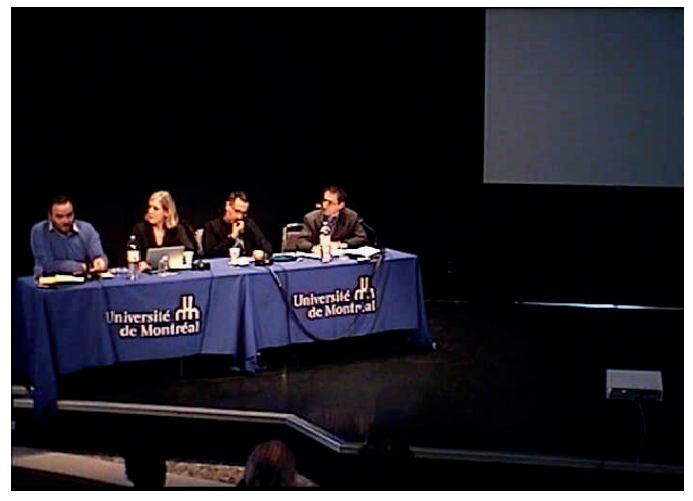

Extrait vidéo 4 : André Péloquin à propos du critique américain Lester Bangs.

Or, il s'avère important de questionner la relation entre une critique musicale et une œuvre : l'une doit-elle être au service de l'autre ? Y a-t-il rapport d'équilibre, de coexistence, ou plutôt de subordination? Chez un auteur comme Berthet, le discours critique joue un rôle dans le processus créateur, « la création artistique et [c]e discours [...] se rencontr[ant] sur le chemin de la création " (Berthet 2006, p. 8). Or, pour les panélistes Machart et Midgette, il semble que l'œuvre musicale conserve une certaine primauté et que leurs critiques relèvent davantage du compte rendu.

En dernier lieu, Duchesneau avait proposé l'enjeu de la crainte du jugement erroné, qui mènerait à une conduite adogmatique. À la lumière des propos de Midgette, ce ne seraient pas tant les critiques musicaux « professionnels » que le grand public qui incarnerait ce phénomène. Elle a noté une difficulté généralisée chez les lecteurs de son blogue à émettre une opinion personnelle vis-à-vis de la musique qu'ils écoutent. Par conséquent, elle a dit encourager son lectorat à prendre la parole et à participer à un débat d'idées, adoptant une approche similaire au philosophe Marc Jimenez pour qui « le jugement de goût marque le point de départ de la réflexion esthétique ; il ne saurait être son aboutissement » (cité par ibid., p. 98-99).

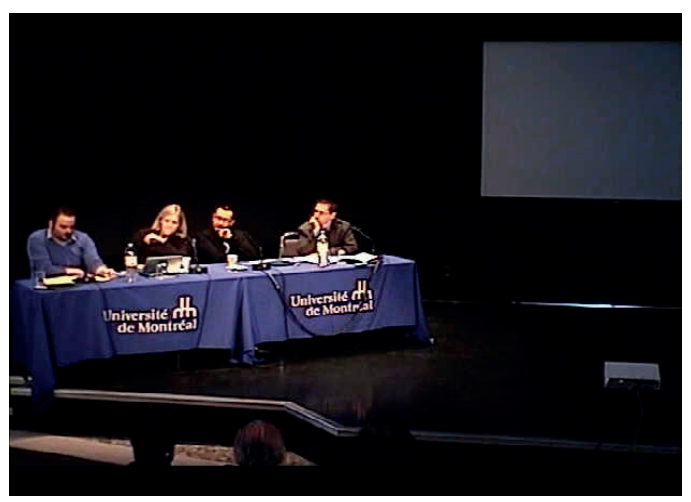

Extrait vidéo 5: Anne Midgette à propos de la participation des lecteurs sur son blogue.

En somme, la question de Duchesneau n'a pas obtenu une réponse directe de la part des panélistes. 
PRoblématiQues tRANSCUlturelles?

Les deux heures réservées à ce panel auront permis de couvrir un large spectre de considérations propres à la pratique de la critique musicale ; nombre des problématiques abordées ont d'ailleurs été reprises dans d'autres conférences du colloque. Il faut par ailleurs souligner que cette séance d'ouverture avait trait à un contexte culturel et spatiotemporel très circonscrit - le contexte occidental contemporain -, tandis que le colloque se voulait un lieu de rencontres entre spécialistes qui s'interrogent sur la notion de goût musical à travers différentes sociétés. Quelques thèmes débattus par les trois critiques musicaux ont néanmoins été revisités dans des conférences portant sur d'autres traditions musicales. C'est notamment le cas de la pertinence sociale de l'œuvre qui aurait préséance sur les considérations d'ordre esthétique. Dans un panel regroupant des ethnomusicologues du Laboratoire de musicologie comparée et anthropologie de la musique (MCAM) de l'Observatoire interdisciplinaire de création et de recherche en musique (OICRM), la professeure Nathalie Fernando soulignait par exemple le peu de recherches ethnomusicologiques portant sur des questions esthétiques dans des sociétés de tradition orale, car les musiques y sont souvent considérées comme étant uniquement fonctionnelles, ce qui a limité les tentatives d'aborder de front les critères du «beau ». Pourtant, les travaux de cette équipe ont su montrer que, malgré le peu de verbalisation dont ils font l'objet, des critères de jugement de goût existent bel et bien dans plusieurs de ces sociétés. Ajoutons que la variabilité du goût, dont a traité le musicologue et sémiologue Jean-Jacques Nattiez lors de sa conférence autour de l' "Essai sur la norme du goût » du philosophe anglais David Hume (1757), n'est pas exclusive à notre contexte sociohistorique ; l'ethnomusicologue Flavia Gervasi, qui travaille sur les chants de la paysannerie dans le Salento, en Italie, a également constaté une hétérogénéité des critères et des conduites sur son propre terrain.

\section{ET L'AVENIR DE LA CRITIQUE?}

Pour terminer, quel avenir prédit-on à la critique musicale ? Chez Midgette, la vision était plutôt sombre : non seulement constatait-elle une diminution de l'espace réservé à la musique classique dans la société, mais elle a aussi déploré un changement d'attitude des journaux qui tenteraient désormais de répondre aux attentes des lecteurs plutôt que de les inciter à la découverte. Par conséquent, elle a émis 1'hypothèse que les postes de critiques musicaux ne cesseront de diminuer. Du côté de Machart, les interrogations étaient nombreuses et, en grande partie, sans réponses. Elles concernaient tant l'identité des lecteurs-auditeurs (à qui s'adresser ?) que la véritable nature d'une réflexion critique sur la musique. Par ailleurs, l'insistance de ce panéliste sur le rôle de mémoire joué par la critique laisse entendre que cette dernière a sa place et doit continuer d'exister, notamment pour les historiens et musicologues de demain. Finalement, Péloquin a considéré que les transformations actuelles de la critique musicale, notamment par la démultiplication des plates-formes de diffusion, induisent une nouvelle dynamique sociale où il y a, selon lui, " hyperdémocratisation " de la pratique. "Everybody's really a critic » a avancé cet invité, donnant ainsi 
raison à Duchesneau qui parlait en introduction d'une ère où « tout est possible, tout est permis".

Le thème de la critique musicale comme telle a ainsi suscité une pluralité de visions et de propositions des panélistes, ce qui n'est certainement pas étranger aux divers contextes - musical, médiatique, mais aussi géographique - au sein desquels ils œuvrent. Or, la question du goût musical a également parcouru cette discussion, apparaissant au cœur des préoccupations des trois participants. Certaines réflexions communes ont émergé à ce compte, en dépit des différents univers musicaux qu'explorent ces trois critiques, ce qui n'est pas sans rappeler l'idée que les clivages seraient de plus en plus flous entre genres musicaux alors qu'une nouvelle figure de la consommation musicale, la figure de 1'« omnivore ${ }^{5}$ ", s'affirmerait désormais.

Enfin, si la critique telle que pratiquée par les trois invités du panel traverse une période houleuse, il ne s'agit pourtant pas de sa première remise en question dans l'histoire (Duchesneau 2007). De fait, les critiques, ces « miroir[s] grossissant[s] des attitudes et des réactions du public en général » selon le sémiologue Jean Molino (2006, p. 1392), ont souvent vu leur rôle et leur raison d'être débattus. Par ailleurs, il importe de ne pas réduire la critique musicale en soi à sa pratique professionnelle, car si la seconde est souvent remise en question, la première, en ce qu'elle « sous-tend la façon dont nous nous expliquons la musique à nous-mêmes » écrit Griffiths (2004, p. 1057), pourra continuer d'exister tant qu'il y aura des auditeurs de la musique.

\section{BIBLIOGRAPHIE}

Bangs, Lester (1988), Psychotic Reactions and Carburetor Dung. The Work of a Legend Critic. New York, Vintage Books.

Bangs, Lester (2003), Main Lines, Blood Feasts, and Bad Taste. A Lester Bangs Reader, New York, Anchor Books.

Berthet, Dominique (2006), Les défis de la critique d'art, Paris, Éditions Kimé.

Duchesneau, Michel (2007), "Critique musicale et esthétique. Pour un projet d'histoire postmoderne», dans Alessandro Arbo (dir.), Perspectives de l'esthétique musicale. Entre théorie et histoire, Paris, L'Harmattan, p. 177-190.

Fernando, Nathalie (2013), « De la variabilité et ou de la similarité des jugements de goût dans les cultures du monde ", conférence présentée au colloque Qu'en est-il du goût musical dans le monde au XXI siècle?, Montréal, Faculté de musique de l'Université de Montréal, $1^{\mathrm{er}}$ mars.

Gervasi, Flavia (2013), «Les logiques du fonctionnement du discours esthétique chez les chanteurs paysans du Salento. Une exégèse inachevée ", conférence présentée au colloque Qu'en est-il du goût musical dans le monde au XXI siècle ?, Montréal, Faculté de musique de l'Université de Montréal, $1^{\text {er }}$ mars.

Griffiths, Paul (2004), « Objectifs et impacts de la critique musicale », dans Jean-Jacques Nattiez (dir.), Musiques. Encyclopédie pour le XXI siècle, «2. Les savoirs musicaux", Arles/Paris, Actes Sud/Cité de la musique, p. 1057-1070.

5 Les travaux du sociologue Richard A. Peterson (voir, entre autres, 1996, 2004) ont initié une série de recherches autour de la question de l'omnivorisme. 
Hume, David ([1757]1974), «La norme du goût », trad. fr. par Renée Bouveresse, Essais esthétiques II. Art et psychologie, Paris, Vrin, p. 79-104.

Kolb, Bonita M. (2000), "You Call This Fun? Reactions of Young First-time Attendees to a Classical Concert ", Journal of the Music and Entertainment Industry Educators Association, http://www.meiea.org/Journal/html ver/Vol01 No01/Vol 1 No 1 Al.html, consulté le 10 novembre 2015.

Kolb, Bonita M. (2001), « The Effect of Generational Change on Classical Music Concert Attendance and Orchestras' Responses in the UK and us ", Cultural Trends, vol. 11, n ${ }^{\circ} 41$, p. 1-35.

Molino, Jean (2006), «Pour une autre histoire de la musique. Les réécritures de l'histoire dans la musique du $\mathrm{xx}^{e}$ siècle ", dans Jean-Jacques Nattiez (dir.), Musiques. Encyclopédie pour le XXI siècle, "4. Histoires des musiques européennes », Arles/Paris, Actes Sud/Cité de la musique, p. 1386-1440.

Nattiez, Jean-Jacques (2013), «Ce que l'essai de Hume sur la norme du goût peut nous apprendre encore aujourd'hui ", conférence présentée au colloque Qu'en est-il du goût musical dans le monde au $X X{ }^{e}$ siècle?, Montréal, Faculté de musique de l'Université de Montréal, $1^{\text {er }}$ mars.

Oliveira-Menezes, Natassja (2015), « Le développement du public jeune universitaire pour la musique classique à Montréal », Mémoire de maîtrise, Université de Montréal.

Peterson, Richard A., et Roger M. Kern (1996), «Changing Highbrow Taste. From Snob to Omnivore», American Sociological Review, vol. 61, n ${ }^{\circ}$ 5, p. 900-907.

Peterson, Richard A. (2004), "Le passage à des goûts omnivores. Notions, faits et perspectives ", Sociologie et sociétés, vol. $36, \mathrm{n}^{\circ}$ 1, p. 145-164. 